\title{
Open Partial Nephrectomy without Ischemia; Case Series
}

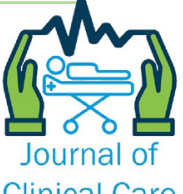

Clinical Care

and Skills

\section{ARTICLE INFO}

\section{Article Type}

Case Series

\section{Authors}

Rabani S.M.*1 PhD

Mousavizadeh A. ${ }^{2} M D, P h D$,

Rabani $S^{3} M D$

\section{How to cite this article}

Rabani S.M, Mousavizadeh A, Rabani S. Open Partial Nephrectomy without Ischemia; Case Series. Journal of Clinical Care and Skills. 2021;2(1):45-49.

\begin{abstract}
A B S T R A C T
Aims Renal vascular clamping during partial nephrectomy can lead to renal ischemia and kidney damage due to reperfusion injuries, but a surgical technique without any ischemia can eliminate such unwanted effects on kidney function. The present study aimed to demonstrate the feasibility of partial nephrectomy in conditions where the renal artery is ready to be clamped.

Patient Information In this case series study, 24 eligible patients with small solitary renal masses were included in the study between 2013 and 2018. The option of surgery was open partial nephrectomy. During the time corresponding to the hilar clamping in standard techniques, the renal artery was exposed and ready for clamping if needed, and in this situation, resecting the mass was carried out.

Findings The mean tumor resection time was $22.08 \pm 2.50$ minutes, and the mean procedure time was $92.00 \pm 4.60$ minutes. Clear cell renal cell carcinoma was seen in 19 patients, papillary renal cell carcinoma in 3 , and oncocytoma in 2 patients. There was no positive surgical margin in specimens with cancer. Lower pole, mid zone, and upper pole masses were observed in 14, 2, and 8 cases, respectively.

Conclusion Although this technique can potentially be a dangerous procedure, in a situation in which the renal artery is well exposed and ready to be controlled, especially when the tumor is in Polar Regions, the mass resection can be safely done without any type of ischemia. Therefore, this procedure can be suggested as a viable one in comparison with other related procedures.
\end{abstract}

Keywords Kidney Neoplasm; Nephrectomy; Ischemia
${ }^{1}$ Beheshti Teaching Hospital, Yasuj University of Medical Sciences, Yasuj, Iran

${ }^{2}$ Social Determinants of Health Research Center, Yasuj University of Medical Sciences, Yasuj, Iran

${ }^{3}$ Cellular and Molecular Research center, Yasuj University of Medical Sciences, Yasuj, Iran

\section{*Correspondence}

Address: Beheshti Teaching Hospital, Yasuj University of Medical Sciences, Yasuj, Iran.

Phone: +98 (74) 33221551

Fax: -

rabani.smr@yums.ac.ir

\section{Article History}

Received: October 25, 2019

Accepted: July 21, 2020

ePublished: February 01, 202

\section{I T A T I O N L I N K S}

[1] Partial versus radical nephrectomy for 4 to $7 \mathrm{~cm}$ renal ... [2] Partial nephrectomy versus radical nephrectomy in patients ... [3] Safety and efficacy of partial nephrectomy for all T1 tumors based ... [4] Renal mass and localized renal cancer: AUA ... [5] Trifecta in partial nephrectomy ... [6] Regional trends of renal mass management ... [7] Partial nephrectomy versus radical nephrectomy for clinically ... [8] Renal ischemia and function after partial nephrectomy ... [9] Tolerance of the human kidney to isolated controlled ... [10] Factors predicting renal functional outcome after partial ... [11] The impact of ischemia time during open nephron sparing ... [12] Every minute counts when the renal hilum is clamped ... [13] The effects of warm ischaemia time on renal function after laparoscopic ... [14] Ischemic renal damage after nephron-sparing surgery in patients ... [15] Assessing the impact of ischaemia time during partial ... [16] Warm ischemia less than 30 minutes is not necessarily ... [17] Laparoscopic versus open partial nephrectomy for stage T1a ... [18] Outcomes of robotic versus laparoscopic partial ... [19] The comparison of perioperative outcomes of robot-assisted ... [20] Systematic review and meta-analysis of comparative studies ... [21] Robotic surgery and hemostatic agents in partial nephrectomy: A high ... [22] Partial nephrectomy: A contemporary review regarding ... [23] Comparison of warm and cold ischemia on renal function ... [24] Comparing zero ischemia laparoscopic radio frequency ... [25] Zero ischemia anatomical partial nephrectomy ... 


\section{Introduction}

Although renal masses can be malignant, benign, or inflammatory, the most common malignant renal mass is renal cell carcinoma (RCC), accounting for 2$3 \%$ of all kinds of adult cancers. Historically the gold standard treatment for RCC was radical nephrectomy (RN), but recent studies suggested that partial nephrectomy (PN); in compared to radical nephrectomy, may be associated with improved survival for small renal tumors (between 4 centimeters (cm) or less and $7 \mathrm{~cm}$ or less), and has the benefit of preserving renal function [1]. Although $\mathrm{RN}$ is a known risk factor of chronic kidney disease (CKD) in patients with renal tumors, it is still the most common procedure which is performed in patients with small renal tumors [2]. PN is mostly used for patients who would be rendered anephric after radical nephrectomy; for example, patients with bilateral RCC and single kidney patients with RCC were PN subjects, and the fear of positive surgical margins leading to an increase the risk of local recurrence, limited the widespread use of PN. The significant improvement in imaging techniques has led to increased detection of small renal tumors and caused PN to be used for these small renal tumors [3]. In patients with small and clinically localized masses, many important factors should be considered for selecting radical nephrectomy, partial nephrectomy, or ablation therapy while counseling the patient themselves. The most important factors are the general health situation and possible associated diseases, the situation of the patient's total renal function, the estimation of the oncological potential of the mass, and finally, the potential morbidity of each management scenario [4]. Selecting each kind of management scenario for small renal masses, the trifecta concept can be used as ideal guidance. The 3 key outcomes of minimal renal function deterioration, no surgical complications, and a negative cancer marker situation can be achieved [5]. Application of Vascular clamp during PN can lead to renal ischemia, and the consequences called reperfusion injuries. Zero ischemia techniques can eliminate adverse impact on renal function (RF).

On a systematic review by Wang Y. and coworkers for evaluating the cost-effectiveness of management options for small renal mass surgeries, ablation was cost-effective versus nephron-sparing surgery, and LPN was cost-effective versus the open approach. However, in contrast, the equipment related procedures are more expensive than open surgeries in our country and other similar countries. On the other hand, Laparoscopic and Robotic surgeries need learning courses, but general urologist can perform partial nephrectomy. Robotic surgery has many limitations in Iran, mostly because of Sanctions. In this complex situation, we have planned a procedure that we think may reach the advantages of 3 key outcomes: minimal renal function deterioration by the technique of no ischemia, less surgical complications by the technique of open surgery, and negative cancer marker that can be achieved by resection of safety margins under direct vision. This procedure will be performed under the exposed renal pedicle's situation, being ready to clamping the renal artery in necessary conditions.

\section{Patient information}

In this case series study in Beheshti teaching Hospital, Yasuj, Iran, 24 patients with small solitary renal masses were recruited to the study between 2013 and 2018. A written consent form was obtained from all participants and stored in their medical records. Patients with solitary renal masses less than $40 \mathrm{~mm}$ and without contraindication to general anesthesia and operation were included in the study, and patients with loss of follow-up and those who did not consent to participate in the study were excluded from the study.

Open partial nephrectomy was performed for patients. This procedure was done in the flank position and through an intercostal, retroperitoneal incision in all patients without removing the rib. A retroperitoneal drain was placed for one day in all patients. Converting the procedure to a nephrectomy was not necessary for this study.

When corresponding to the hilar vessels clamping in standard procedures, we reached the renal artery and were ready to clamp if needed, and in this situation, resection of the mass was assessed. All patients underwent the procedure with 1 to $2 \mathrm{~cm}$ of free margins. All of the patients were evaluated by abdominal spiral CT scan without and with intravenous contrast for defining the exact size and location of the renal masses. All patients were evaluated by abdominal spiral CT scan without and with intravenous contrast to define the renal masses' exact size and location.

The variables such as tumor size, mean tumor resection time, mean operation time, mean estimated blood loss, the possibility of transfusion, frequency of tumor type, site of the tumor in the kidney, length of hospital stay (days), serum creatinine level before the operation and at the time of discharge were gathered by the written form.

Data were analyzed using SPSS 21 software. Descriptive statistics such as mean, standard deviation, frequency, and percent were applied for data analysis. Comparison of serum creatinine levels in patients before and after surgery was performed using paired t-test.

\section{Findings}

The mean age of patients was $41.96 \pm 4.94$ (range 3757) years old. Demographic and clinical characteristics of patients undergoing surgery have been presented in Tables 1 and 2). 
Serum creatinine levels were measured daily, which remained normal until the day of discharge (median 5 days, range 4-7 days), and there was no statistically significant difference before and after surgery (Table 3).

Table 1) Frequency distribution of demographic and clinical characteristics of patients undergoing surgery $(\mathrm{n}=24)$

\begin{tabular}{lcc}
\multicolumn{1}{c}{ Variables } & Number & Percent \\
\hline Sex & & \\
Female & 8 & 33.3 \\
Male & 16 & 66.7 \\
Side & & \\
Right & 11 & 45.8 \\
Left & 13 & 54.2 \\
Tumor site & & \\
Lower pole & 14 & 58.3 \\
Mid zone & 2 & 8.3 \\
Upper pole & 8 & 33.3 \\
Complications & & \\
No complication & 13 & 54.2 \\
Fever & 5 & 20.8 \\
Knee pain & 2 & 8.3 \\
Fever + pain & 4 & 16.7 \\
Pathologic type of tumors & \\
Clear cell RCC & 19 & 79.2 \\
Papillary RCC & 3 & 12.5 \\
Oncocytoma & 2 & 8.3 \\
\hline
\end{tabular}

Table 2) Mean clinical characteristics of patients undergoing surgery $(n=24)$

\begin{tabular}{lcc}
\hline \multicolumn{1}{c}{ Variables } & Min-Max & Mean \pm SD \\
\hline Tumor size $(\mathrm{mm})$ & $22-39$ & $31.92 \pm 4.39$ \\
Procedure time (min) & $72-128$ & $92.00 \pm 4.60$ \\
Tumor resection time (min) & $18-28$ & 22.082 .50 \\
Estimated blood loss ${ }^{*}(\mathrm{ml})$ & $200-500$ & $300.00 \pm 62.00$ \\
Length of hospital stay (days) & $2-7$ & $5.00 \pm 1.35$ \\
\hline
\end{tabular}

${ }^{*}$ With no case of transfusion

Table 3) Comparison of serum creatinine levels in patients before and after surgery using paired t-test

\begin{tabular}{lccc}
\hline \multicolumn{1}{c}{ stage } & $\begin{array}{c}\text { Serum } \\
\text { creatinine }\end{array}$ & $\begin{array}{c}\text { Mean } \\
\text { difference }\end{array}$ & p.value \\
\hline Before surgery & $1.227 \pm 0.28$ & 0.287 & 0.7 \\
After surgery & $1.198 \pm 0.23$ & & \\
\hline
\end{tabular}

\section{Discussion}

The current study has been designed for evaluating the feasibility of open partial nephrectomy without cold or warm ischemia but in a setting of the exposed renal pedicle and ready for possible unexpected events. Today PN is an accepted procedure for small renal malignant tumors. However, there are many options for performing this procedure according to the surgeon's preference, patient situation, availability of surgical equipment, and ablative procedures; open, laparoscopic, and robotic surgery may be selected. At present, it emphasizes conducting a nephron sparing technique for managing renal tumors due to similar oncogenic results while potentially reducing renal and cardiovascular
Rabani S.M. et al.

morbidity compared to RN [6]. While open RN has been the gold standard procedure for renal tumor operation, PN is currently utilized for renal tumor surgery in high and low volume urological centers worldwide. Many previous studies have revealed better overall survival in patients who undergo PN than RN and have shown similar oncological outcomes [7].

Although PN is the gold standard treatment for localized and small renal tumors, there is no consent over the effects of duration and type of intraoperative ischemia on renal function after PN [8]. To evaluate renal function after $\mathrm{PN}$ or $\mathrm{RN}$, serum $\mathrm{Cr}$ is measured; although this test is not a reliable indicator of renal function, it is currently the most commonly used and the easiest test for assessing renal function after partial nephrectomy. Acute ischemia's pathogenesis on the renal function that causes acute kidney damage is based on vascular events, obstructive events, and reperfusion injuries. During these three inevitable processes, persistent vasoconstriction and the abnormal response of endothelial cells, formation of casts by sloughed tubular epithelial cells in the kidney and membrane debris that obstruct tubules, and finally generation of reactive oxygen species, hypercoagulation states, cellular derangement, and microvessel congestion and compression can significantly reduce renal blood flow. On the other hand, many preoperative factors can influence postoperative renal function. Age, sex, tumor size, presence of solitary functional kidney, ischemia type, duration of ischemia, amount of preserved functional kidney, and kind of surgical intervention are among these effective factors. The optimum time of ischemia during PN has not been determined yet, but warm ischemia time (WIT) less than 30 minutes historically thought to allow complete recovery of kidney function. Despite this data, in a human study in 40 patients who underwent open partial nephrectomy with WIT for more than 30 minutes, a greater than expected resistance to ischemia time was shown [9]. Overall, according to several studies, 20 to 25 minutes duration of warm ischemia represents the most accurate cut-off to separate patients who do and do not develop short and long-term renal function decline after PN [10-16],

In a clinical trial by Rezaeetalab and coworkers for comparing laparoscopic (LPN) versus open partial nephrectomy (OPN), for small renal tumors, they have shown that LPN has some benefits over OPN, including decreasing postoperative pain and higher patient satisfaction, but have suggested the higher possibility of positive tumor margin and urinary leakage in LPN [17]. This study correlates with our study at the point of no complication.

Leow et al. meta-analysis for comparing outcomes of robotic partial nephrectomy (RPN) versus LPN demonstrated that RPL confers a superior morbidity profile compared to LPN in most studies [18]. We did not have any significant morbidity in our study. 
Open Partial Nephrectomy without Ischemia; Case Series

In 2 consecutive systematic reviews and metaanalyses by Zhonghua Shen and coworkers and Xia L. and coworkers for comparing perioperative outcomes of robot-assisted partial nephrectomy (RAPN) and OPN, demonstrated that RAPN offered reduced perioperative complications, lesser blood loss, and shorter period of hospital admission than OPN, suggesting that RAPN may be a useful alternative to OPN $[19,20]$. We did not have availability for robotic surgery, and regarding the cost of robotic surgery, it may have a negative point in our center.

We did not have any technical challenges in our clinical trial. Morelli L. and coworkers in a clinical trial using Robotic surgery and hemostatic agents in partial nephrectomy lead to a higher rate of success $(82 \%)$ without vascular clamping and suggested RAPN as a technique overcome technical challenges of LPN [21].

Nowadays, open PN is evolving as the standard of care for managing all amendable renal tumors with laparoscopic, robotic assisted surgery has been widely used [22]. Regarding better preservation of renal function in OPN, in comparison to $\mathrm{RN}$, this technique seems to have a major benefit. LPN seems to be associated with a longer ischemia time, a higher re-operative rate, and increased complication rates. Currently, surgeons have main concerns over preserving renal function in any operative techniques with continued efforts toward decreasing warm ischemia without compromising the oncological efficacy ${ }^{[22] . ~ O u r ~ s t u d y ~ o m i t t e d ~ a n y ~ t y p e ~ o f ~ i s c h e m i a ~ i n ~}$ the setting of exposed renal artery, ready for clamping, which means a lower risk of ischemia in preserved, functioning, and healthy renal tissue.

Funahashi Y. and coworkers compared warm and cold ischemia on renal function after $\mathrm{PN}$ and reported that warm ischemia for more than 25 minutes caused widespread injuries to the operated kidney. In contrast, cold ischemia for less than 58 minutes prevented ischemic injury to the kidney's preserved part [23]. We did not use any type of ischemia; thus, no ischemia-induced complication could be considered. A study conducted by Jiwei Huang and coworkers found that in comparison to conventional LPN, by considering the safety and efficacy of laparoscopic radiofrequency ablation (LRFA) during a prospective randomized controlled trial, LRFA assisted tumor enucleation with no ischemia, enables tumor excision with better renal function preservation [24]. This clinical trial confirms the feasibility and safety of our study. Inderbir S.Gill and coworkers' results about the concept of zero ischemia were similar to our study, although they had different types of surgical interventions [25].

We have omitted any type of ischemia in the exposed renal artery setting in our surgical technique and showed that this technique might be feasible. We suggest more studies with a higher number of patients to support this study's results and designing the randomized clinical trial.

\section{Conclusion}

Current renal tumor management focuses on nephron-sparing techniques due to equivalent oncogenic results while potentially decreasing renal and cardiovascular morbidity compared to radical nephrectomy. Every type of ischemia may have the potential of developing short- and long-term renal function decline after partial nephrectomy. However, in our surgery technique, we have omitted any type of ischemia in the exposed renal artery, ready for clamping, which means a lower risk of ischemia in preserved, functioning, and healthy renal tissue. On the other hand, in our study, OPN is a significantly cost-effective procedure with no need for expensive equipment.

Acknowledgments: The authors thank the director of Beheshti university hospital for a good facility to reach the participants' medical records.

Ethical Permissions: All procedures performed in this study were under the institutional and/or national research committee's ethical standards with the 1964 Helsinki declaration and its later amendments or comparable ethical standards. Ethics Committee approved this study of Yasuj University of Medical Sciences (P.23/2/1411/08/10/2015). A written consent form was obtained from all participants and stored in their medical records.

Conflict of Interests: The authors declare they have no conflict of interest.

Authors' Contributions: Rabani S.M. (First author), Introduction author/Original researcher/Discussion author (60\%); Mousavizadeh A. (Second author), Methodologist/Statistical analyst (20\%); Rabani S. (Third author), Introduction author/Discussion author (20\%).

Funding/Sources: None Declared.

\section{References}

1- Thompson RH, Siddiqui S, Lohse CM, Leibovich BC, Russo $P$, Blute ML. Partial versus radical nephrectomy for 4 to 7 cm renal cortical tumors. J Urol. 2009;182(6):2601-6.

2- Huang WC, Elkin EB, Levey AS, Jang TL, Russo P. Partial nephrectomy versus radical nephrectomy in patients with small renal tumors-is there a difference in mortality and cardiovascular outcomes?. J Urol. 2009;181(1):55-61.

3- Patard JJ, Shvarts O, Lam JS, Pantuck AJ, Kim HL, Ficarra $\mathrm{V}$, et al. safety and efficacy of partial nephrectomy for all T1 tumors based on an international multicenter experience. J Urol. 2004;171(6 Pt 1):2181-5.

4- Campbell S, Uzzo RG, Allaf ME, Bass EB, Cadeddu JA, Chang A, et al. Renal mass and localized renal cancer: AUA guideline. J Urol. 2017;198(3):520-9.

5- Hung AJ, Cai J, Simmons MN, Gill IS. Trifecta in partial nephrectomy. J Urol. 2013;189(1):36-42.

6- Hammett J, Crispen PL, Ko J, Byrd N, Bylund JR, Schenkman NS, et al. Regional trends of renal mass management in the southeast. J Clin Oncol. 2011;29(15):16562.

7- Kunath F, Schmidt S, Krabbe LM, Miernik A, Dahm P, Cleves A, et al. Partial nephrectomy versus radical 


\section{9}

nephrectomy for clinically localized renal masses. Cochrane Database Syst Rev. 2017;5(5):012045.

8- Volpe A, Blute ML, Ficarra V, Gill IS, Kutikov A, Porpiglia $F$, et al. Renal ischemia and function after partial nephrectomy: A collaborative review of the literature. Eur Urol. 2015;68(1):61-74.

9- Parekh DJ, Weinberg JM, Ercole B, Torkko KC, Hilton W, Bennett $\mathrm{M}$, et al. Tolerance of the human kidney to isolated controlled ischemia. J Am Soc Nephrol. 2013;24(3):506-17.

10- Lane BR, Babineau DC, Poggio ED, Weight CJ, Larson BT, Gill IS, et al. Factors predicting renal functional outcome after partial nephrectomy. J Urol. 2008;180(6):2363-9.

11- Thompson RH, Frank I, Lohse CM, Saad IR, Fergany A, Zincke $\mathrm{H}$, et al. The impact of ischemia time during open nephron sparing surgery on solitary kidneys: A multiinstitutional study. J Urol. 2007;177(2):471-6.

12- Thompson RH, Lane BR, Lohse CM, Leibovich BC, Fergany A, Frank I, et al. Every minute counts when the renal hilum is clamped during partial nephrectomy. Eur Urol. 2010;58(3):340-5.

13- Porpiglia F, Fiori C, Bertolo R, Angusti T, Piccoli GB, Podio V, et al. The effects of warm ischaemia time on renal function after laparoscopic partial nephrectomy in patients with normal contralateral kidney. World J Urol. 2012;30(2):257-63.

14- Funahashi Y, Hattori R, Yamamoto T, Kamihira O, Kato $\mathrm{K}$, Gotoh M. Ischemic renal damage after nephron-sparing surgery in patients with normal contralateral kidney. Eur Urol. 2009;55(1):209-15.

15- Becker F, Van Poppel H, Hakenberg OW, Stief C, Gill I, Guazzoni G, et al. Assessing the impact of ischaemia time during partial nephrectomy. Eur Urol. 2009;56(4):625-35. 16- Patel AR, Eggener SE. Warm ischemia less than 30 minutes is not necessarily safe during partial nephrectomy: Every minute matters. Urol Oncol. 2011;29(6):826-8.
Rabani S.M. et al.

17- Rezaeetalab GH, Karami H, Dadkhah F, Simforoosh N, Shakhssalim N. Laparoscopic versus open partial nephrectomy for stage $\mathrm{T} 1 \mathrm{a}$ of renal tumors. Urol J. 2016;13(6):2903-7. [Persian]

18- Leow JJ, Heah NH, Chang SL, Chong YL, Png KS. Outcomes of robotic versus laparoscopic partial nephrectomy: An updated meta-analysis of 4,919 patients. J Urol. 2016;196(5):1371-7.

19- Shen Z, Xie L, Xie W, Hu H, Chen T, Xing C, et al. The comparison of perioperative outcomes of robot-assisted and open partial nephrectomy: A systematic review and meta-analysis. World J Surg Oncol. 2016;14(1):220.

20- Xia L, Wang X, Xu T, Guzzo TJ. Systematic review and meta-analysis of comparative studies reporting perioperative outcomes of robot-assisted partial nephrectomy versus open partial nephrectomy. J Endourol. 2017;31(9):893-909.

21- Morelli L, Morelli J, Palmeri M, Disidoro C, Kauffmann EF, Tartaglia D, et al. Robotic surgery and hemostatic agents in partial nephrectomy: A high rate of success without vascular clamping. J Robot Surg. 2015;9(3):215-22.

22- Riggs SB, Larochelle JC, Belldegrun AS. Partial nephrectomy: A contemporary review regarding outcomes and different techniques. Cancer J. 2008;14(5):302-7.

23- Funahashi Y, Yoshino Y, Sassa N, Matsukawa Y, Takai S, Gotoh M. Comparison of warm and cold ischemia on renal function after partial nephrectomy. Urology. 2014;84(6):1408-12.

24- Huang J, Zhang J, Wang Y, Kong W, Xue W, Liu D, et al. Comparing zero ischemia laparoscopic radio frequency ablation assisted tumor enucleation and laparoscopic partial nephrectomy for clinical t1a renal tumor: A randomized clinical trial. J Urol. 2016;195(6):1677-83.

25- Gill IS, Patil MB, De Castro Abreu AL, Ng C, Cai J, Berger A, et al. Zero ischemia anatomical partial nephrectomy: A novel approach. J Urol. 2012;187(3):807-14. 